\title{
Success factors for deploying cloud computing in eHealth
}

\author{
Nermeen Mekawie ${ }^{1}$, Kesmat Yehia $^{2}$ \\ ${ }^{1}$ Assistant Professor, Head of Business Information Systems Department, Arab Academy for Science, Technology and Maritime \\ Transport, 2033, El Horia, Cairo, Egypt \\ ${ }^{2}$ Assitant Lecturer, Business Information Systems Department, Arab Academy for Science, Technology and Maritime Transport, 2033, \\ El Horia, Cairo, Egypt
}

\begin{abstract}
Developing countries different sectors especially healthcare suffers from redundancy, lack of communication, lack of information, lack of resources, lack of sharing and lack of supervision as well as diagnosis errors. Cloud computing may offer great opportunities for the developing countries to modernize the healthcare sector and hence overcome the problems it suffers. Adoption of the cloud computing poses different challenges and obstacles which may complicate the actual implementation. This study aims at investigating the success factors for deploying cloud computing within the eHealth sector in developing countries and consequently the study employed the case of Egypt as a developing country. This research tested the critical factors quantitatively which was embedded within critical success factors models adopted from the literature within the context of cloud computing implementation. The model includes all the critical success factors and the effect of demographics on the factors for a successful implementation of cloud computing in the healthcare industry.
\end{abstract}

\section{Introduction}

Although technology has been a powerful tool for poverty reduction and improved standards of living in developing countries, the benefits have not been evenly distributed across the globe. Therefore, a great potential lies in the transfer of technologies to those areas that are still yet to benefit from such innovations and technological progress (Haliu, 2012). Thus, studying the cloud computing implementation in Egypt as a developing country can be of great help to the modernization of eHealth sector in the developing countries. Besides, they can cope with the west and enhance the availability of health care services with lower costs.

A number of researchers aimed at studying the factors responsible for the technology adoption in developing countries in quest of modernization. Numerous studies were conducted in the context of exploring factors for cloud computing adoption. Wahid (2007) and Atsu, Andoh-Baidoo, Osatuyi, and AmoakoGyampah (2010) applied the adoption theory to research Internet adoption in Indonesia and Ghana respectively (Wahid, 2007). Also, Alemayehu Hailu in 2012 studied the cloud computing acceptance in developing countries. Shahid Al Noor, Golam Mustafa, Shaiful Alam Chowdhury, Md. Zakir Hossain, Fariha Tasmin Jaigirdar in 2010 studied the cloud computing in education in Bangladesh. Although there are a lot of researches discussing the success factors and the acceptance of cloud computing, there is a lack of literature concerning the developing countries (Shahid Al Noor, 2010). Adoption and implementation of a new technology are always complex and is done through stages. Researchers tried to discuss the subject of adoption and implementation of technologies in developing countries. Wahid (2007) analysed Internet adoption and usage among men and women in Indonesia. (Riyadh et al., 2009) conducted a research exploring the factors that influence the adoption of e-banking in developing countries as well (Riyadh, Akter , \& Islam, 2009). Atsu (2010) also performed a case study of information and communication technology (ICT) project implementation within a company in Ghana to highlight factors that underlie the implementation of ICT projects and how these factors collectively impact project success (Atsu, 2010).

Developing countries lack resources, infrastructure and different elements of successful implementation of new technologies. Kshetri (2010) attested that cloud computing allows access to advanced IT infrastructure, data centres, and applications that most developing countries do not have. Based on the review and study of the literature, the gap about the factors needed for successful implementation of cloud computing in eHealth in developing countries should be examined. The researcher chose the case of Egypt as a developing country and the information gained will provide insight that will help in the successful implementation of cloud computing in eHealth in Egypt. Cloud computing in developing countries can be seen as an opportunity to solve some problems in different sectors and modernizing them at the same time.

Cloud computing can be used to modernize the eHealth sector in Egypt as a developing country and hence enhance the healthcare service delivery. Hospitals

\footnotetext{
* Corresponding author: nermeen.magdi@aast.edu
} 
will be able to use the cloud as a public utility to pay per use model. Studying the literature review, a gap was found regarding the factors which influence the adoption and implementation of cloud computing in developing countries. Besides the benefits the cloud offers for modernizing the eHealth sector within the developing countries. Cloud computing poses a drastic opportunity for developing countries in eHealth such as scalability, sharing, collaboration, access to more information resources, research mining, back up, interactive multimedia learning environments and cutting costs.

This study contributes to the body of knowledge by studying the success factors for cloud computing deployment in eHealth within Egypt as a developing country. Subsequently the study tries to fill the literature gap found and accordingly help in the successful implementation of cloud computing in developing countries to modernize the healthcare sector.

- A proposed model for the success factors of cloud computing deployment of eHealth.

- The challenges facing the cloud implementation in eHealth

- The opportunities which the cloud offers to the healthcare sector

- Demographics and their effects on the proposed factors.

\subsection{Related Work}

The concept of critical success factors (CSF) was introduced by Rokhart in 1979 to explain a number of areas in which desirable results will ensure successful competitive performance for an organization or areas for the organization to flourish. Over the past decade, studies have been undertaken to study the CSFs for different IT systems and initiatives (Al-Mashari, 1999) (Wixom \& Watson, 2001); (Poon, 2001). Cloud computing, however differs from IT initiatives because it exceeds the boundary of intra-organization matter. Cloud computing also is considered as an innovation and requires change in business processes. Different researchers studied the critical factors for cloud computing adoption and each one reached different results with similarities.

According to Omotude, Awodele, Kuyoro and Ajaegbu in 2009, The cloud computing technology requires various factors for successful implementation which they categorized as (Omotude , 2009):

-Ability to access and manage risks

-Performance

-Security and privacy

-Trust

-Financial issues

Haliu in 2012 studied the success factors for cloud computing implementation from the managers' perspective or IT leaders in developing countries. He used quantitative methods of online surveys. The study utilized a cross-sectional survey with 16 questions. The tested success factors were:

- Cost effectiveness of cloud computing

-Cloud computing reliability

-Security

-The need for cloud computing

Garrison in 2012 researching the key factors for cloud computing deployment. Garrison concluded that trust, technical capability and managerial capability are the three factors affecting the deployment positively. The study used questionnaire which didn't fit since the topic is still nascent as mentioned before. It is not right to assume that the factors or constructs identified from traditional IT initiatives could apply to the new context of cloud computing deployment. In 2013, Shuguang Suo submitted a dissertation regarding the critical success factors, challenges, and impacts of cloud computing on the IT function. Ezer Osei in 2014 examined the factors influencing the cloud computing adoption by small and medium sized companies in developing economies. Selecting Ghana as a case study and using quantitative analysis only, the following factors were concluded.

-Technological factors like Trainability of cloud services, existence of required IT infrastructure, Compatibility with existing system, Strength of In-built Security systems, Learning Capability of employees, Limited technical knowledge about similar technologies and nonperformance of cloud services to support operations.

- Organizational factors like Top management support, Resistance towards new technologies, Conformity with work culture and style, impact of organizational structure and size and first adopters in our industry.

-Environmental factors like adequate user and technical support from provider, choice of skilled employees, influence of market scope, the nature of the industry

In 2014, Liezel Cillers studied using the cloud to provide telemedicine services in South Africa and concluded the following success factors:

-National integrated plan for telemedicine.

-Best practices within a legislation framework

-Involve the end user

-Provide education to improve the level of awareness

-Address technology issues

\subsection{Proposed Research Model}

The figure shows the proposed research model for the dissertation which investigates the success factors for cloud computing adoption and deployment in eHealth in 
developing countries (case of Egypt). The ovals represent the broad or generic factors which each contains a number of sub factors to be examined. Each of the generic factors influences the adoption in positive, negative or neutral way. Each relation is represented in the hypotheses and tested through the collection and analysis phases. Each factor was chosen based on a study of the literature of critical success factors of IT initiatives and especially cloud computing.

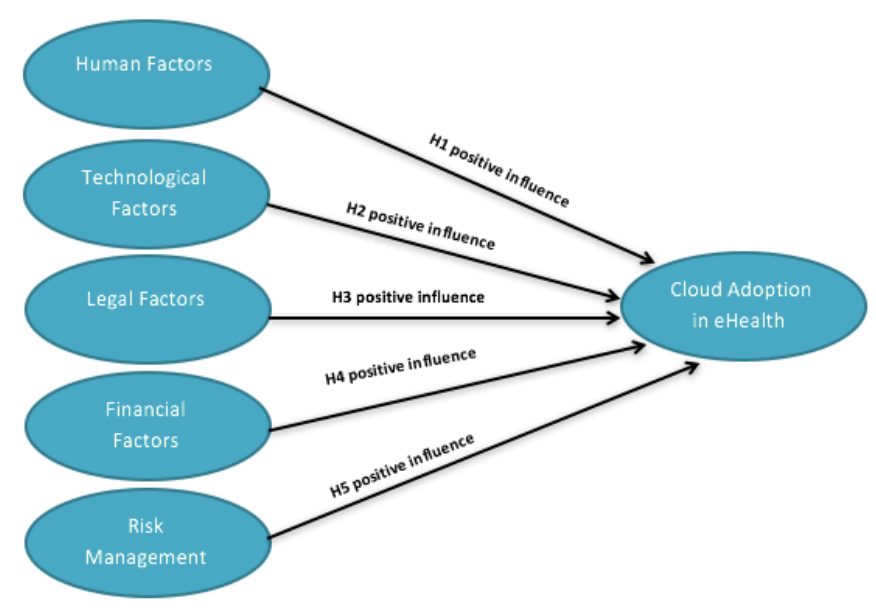

Fig. 1. Proposed Framework

\subsection{Hypotheses}

The human factors encompass factors relevant to human involvement in the adoption of cloud computing. The human factors are studied since the human involvement is very important in the successful implementation of any IT initiatives as mentioned in the literature of CSFs.Sub factors of the human factors include: Management support, Healthcare actors support.

\section{Hypothesis 1}

Human factors may influence the adoption of cloud computing in eHealth positively.

The technological factors contain factors regarding the technological infrastructure, architecture. Sub factors of technological factors are Organizational Infrastructure, Security, Ease of use and Service Quality.

\section{Hypothesis 2}

Technological factors may influence the adoption of cloud computing in eHealth positively.

Legal and regulatory factors included factors of regulatory compliance and agreements like SLA and country laws. This is a very risky dimension since people won't trust dealing with cloud computing unless there are strict laws and compliance to protect their data against any breach. Legal and regulatory sub factors are Regulations and laws availability, Compliance with regulations, Transparency of cloud services

Hypothesis 3

Legal and regulatory factors may influence the cloud computing adoption positively.

Financial factors are important, especially in developing countries where the limited resources is the predominant status. Hence the low initial investment cost with return on investment is an important incentive to adopt cloud computing in eHealth. The financial factors include the cost of deploying cloud computing.

Hypothesis 4

Financial factors may influence the cloud computing adoption positively.

Risk management is also important to examine since the data on the eHealth cloud will be sensitive data and requires availability and sustainability. Therefore, any risk that might be faced, risk management plans should be designed proactively like data risks, security risks, support risk, influence of industry and political or economic risks.

Hypothesis 5 
Risk management may influence the cloud computing adoption positively.

\section{Research Methodology}

The study employed the mixed methodology method. The first step towards the results was to conduct the interviews to explore the nascent nature of the topic from different dimensions. Interviews were conducted until information saturation was achieved in an attempt to explore the problem under study besides the intensive literature reviewed and studied. Therefore, interviews were conducted with cloud experts, doctors and hospital managers to understand the underlying relationships of variables. The interviews were analyzed first individually and each interview analysis will be presented in details with quotes from the interviewee to support the analysis. Then cross analysis was carried out to find the similarities and differences to reach confirmed results, whether there are variables effect or not. Questionnaires were distributed randomly through two channels; online and hardcopy. The online questionnaire used "Kwik survey". The questionnaire targeted doctors with different demographic features and different work experiences and specialties. Hardcopies were distributed to doctors in both private and public hospitals with the help of family, relatives and connections. Some questionnaires were self-completed while others requested face to face questionnaire.

\section{Conclusion}

After calculating the $\mathrm{p}$-value for every factor through the hypothesis test, all p-values were less than 0.05 indicating an influential relationship between the factors and cloud computing adopting in eHealth within Egypt. The results are demonstrated on the following figure to indicate the strong influence between each factor and cloud adoption.

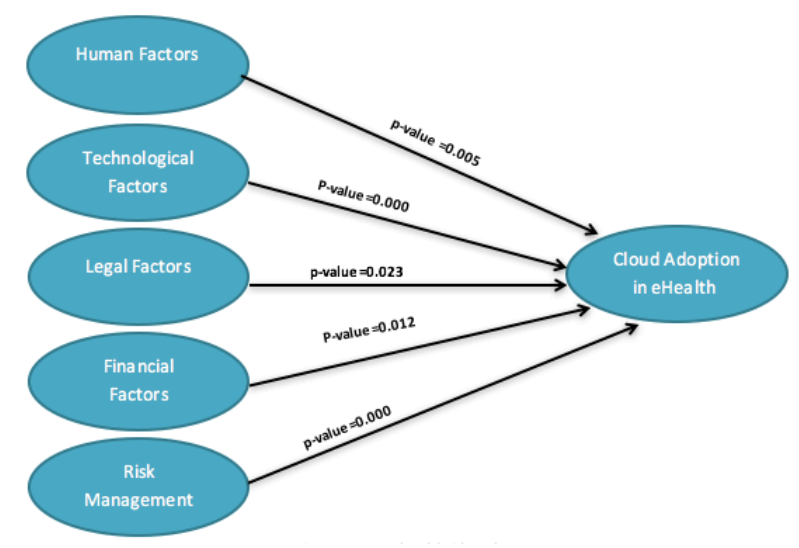

Fig. 2.Framework with $\mathrm{p}$ Value

The p-value computed for Human Factors was 0.005 which is less than the p-value tabulated 0.05 which indicates a significant influence on cloud adoption in eHealth. For Technological factors, the p-value was 0.000 which is less than p-value tabulated 0.05 indicating a strong influence on cloud adoption. Whereas the $p$-value for legal factors is 0.023 indicating an influence over the adoption. Financial Factors had pvalue of 0.012 which shows a strong influence on adoption and risk management had a p-value of 0.000 indicating a significant influence. Another aspect which was studied is the effect of demographics on the factors. The effect was test using the t-test and ANNOVA. The following figure shows the effect of demographics on the factors.

The results obtained from the data analysis of both quantitative and qualitative data shows that all factors proposed influence the adoption of cloud computing in eHealth in developing countries.

According to the analysis, it was found that all the factors are important, however some factors tend to be more important. The factors according to importance were:
-Human factors
- Financial factors
- Technological factors
-Legal factors
-Risk Management

A significant relationship was found between all the factors. Another aspect which was studied is the effect of demographics on the factors. The gender, for example and educational level proved to have an insignificant relationship with any factor. However, age and experience influenced the human and technological factors. Position and sector influenced legal and financial factors. Finally, eHealth and cloud awareness influenced the human factors only.

\section{Future Work}

There are numerous recommendations for future research. Expanding the scope of the study to include other geographical areas like different developing countries or rural and urban areas in the same country. This will consequently open the door towards 
comparative studies to examine how the demographics influence the success factors deeply. With the global nature of computing, research could be performed considering international cloud computing issues. Also evaluating the success factors in different sectors like business, education and so on to study the differences of the cloud computing success factors in different sectors within the same context of developing countries. This will enlighten the decision makers such as the government if considering offering cloud solutions for the different sectors within the country. Another future work may be adding other factors to examine which may be derived from other research contexts to uncover hidden factors which may influence the cloud adoption in eHealth in developing countries. Finally, studying the culture of the population and how it influences the adoption of the cloud in eHealth is very important. The population, culture in developing countries tends to fear any services offered from the government that require them to submit personal information. Thus, a sociocultural study should be conducted to further examine the culture of the developing countries and the ways to deal with it and heal the injured pieces of culture to be able to successfully implement the technology and cope with the era they live in.

\section{References}

[1] Haliu, factors influencing cloudcomputing technology adoption in developing countries, 2012.

[2] F. Wahid, "Using the technology adoption model to analyze Internet adoption and use among men and women in Indonesia," The Electronic Journal on Information Systems in Developing Countries, pp. 1-8, 2007.

[3] Shahid Al Noor, "A Proposed Architecture of Cloud Computing for Education System in Bangladesh and the Impact on Current Education System," International Journal of Computer Science and Network Securit, 2010.

[4] A. Riyadh, M. Akter and N. Islam, "The adoption of e-banking in developing countries: A theoretical model for
SMEs," International Review of Business Research Papers, pp. 212-230., 2009.

[5] Atsu, "an exploratory study of the contextual factors that influence success of ict projects in developing nations: a case study of a telecommunications company in ghana," Journal of Information Technology Case \& Application Research, 2010.

[6] Al-Mashari, "BPR implementation process: an analysis of key success and failure factors," Business Process Management Journal, 1999.

[7] B. Wixom and H. Watson, "An empirical investigation of the factors affecting data warehousing succes," MIS Quarterly, 2001.

[8] P. Poon, "Critical success factors revisited: success and failure cases of information systems for senior executive," Decision Support Systems, 2001.

[9] Omotude, "Survey of Cloud Computing Issues at Implementation Level," Journal of Emerging Trends in Computing and Information Sciences, 2009. 